\title{
An Ultra-fast Linear Array Detector for MHz Line Repetition Rate Spectroscopy
}

\author{
L. Rota*, M. Balzer*, M. Caselle*, S. Kudella*, M. Weber*, A. Mozzanica ${ }^{\dagger}$, N. Hiller ${ }^{\dagger}$, M.J. Nasse ${ }^{\ddagger}$, G. Niehues ${ }^{\ddagger}$, \\ P. Schönfeldt ${ }^{\ddagger}$, C. Gerth ${ }^{\S}$, B. Steffen ${ }^{\S}$, S. Walther ${ }^{\S}$, D. Makowski**, A. Mielczarek ${ }^{* *}$
}

\begin{abstract}
We developed a fast linear array detector to improve the acquisition rate and the resolution of Electro-Optical Spectral Decoding (EOSD) experimental setups currently installed at several light sources. The system consists of a detector board, an FPGA readout board and a high-throughput data link. InGaAs or Si sensors are used to detect near-infrared (NIR) or visible light. The data acquisition, the operation of the detector board and its synchronization with synchrotron machines are handled by the FPGA. The readout architecture is based on a high-throughput PCI-Express data link. In this paper we describe the system and we present preliminary measurements taken at the ANKA storage ring. A line-rate of $2.7 \mathrm{Mlps}$ (lines per second) has been demonstrated.
\end{abstract}

Index Terms-High Speed Linear Array Detector, Beam Monitoring, FPGA, Readout Electronics.

\section{INTRODUCTION}

D URING the past decade, Electro-Optical Spectral Decoding (EOSD) emerged as a promising technique to measure the longitudinal bunch profile in a non-destructive way. Sub-picosecond spatial resolutions have been achieved [1], therefore making EOSD of high interest for VUV and Xray FELs. Several accelerator machines have commissioned beam diagnostic stations based on EOSD [1]-[4].

A description of the EOSD technique can be found in [5]. In short, this technique allows to reconstruct the bunch profile by measuring the spectrum of a laser pulse, which has been modulated inside an electro-optical crystal by the Coulomb field of the charged beam. A spectrometer equipped with a linear array detector is typically used to measure such modulation and reconstruct the bunch profile. As opposed to previous methods, which allowed only averaged measurements over more bunches [6], the EOSD technique allows measurements on a single-shot basis. However, in order to fully take advantage of the single-shot resolution, the acquisition rate of the spectrometer must match the bunch repetition rate (e.g.: $2.7 \mathrm{MHz}$ at ANKA with single-bunch filling, $4.5 \mathrm{MHz}$ at XFEL). The main bottleneck in current setups lies in the line rate of commercial line cameras, with the fastest ones reaching only hundreds of $\mathrm{kHz}$ [7], [8].

In order to increase the acquisition rate of EOSD experiments, we have developed a linear array detector with a

Author's contact: lorenzo.rota@kit.edu

* Institute for Data Processing and Electronics (IPE), Karlsruhe Institute of Technology, Karlsruhe, Germany

$\dagger$ Paul Scherrer Institute (PSI), Villigen, Switzerland

$\ddagger$ LAS and IBPT, Karlsruhe Institute of Technology, Karlsruhe, Germany

$\S$ Deutsches Elektronen-Synchrotron (DESY), Hamburg, Germany

** Lodz University of Technology, Lodz, Poland line-rate of $2.7 \times 10^{6} \mathrm{lps}$ (lines per second). In this paper we describe the detector card and the readout architecture developed for the experimental setup at ANKA.

\section{Architecture}

The system consists of a detector card and an FPGA-based readout card, connected together through an FMC connector [9]. A block diagram of the detector's architecture is shown in Figure 1.

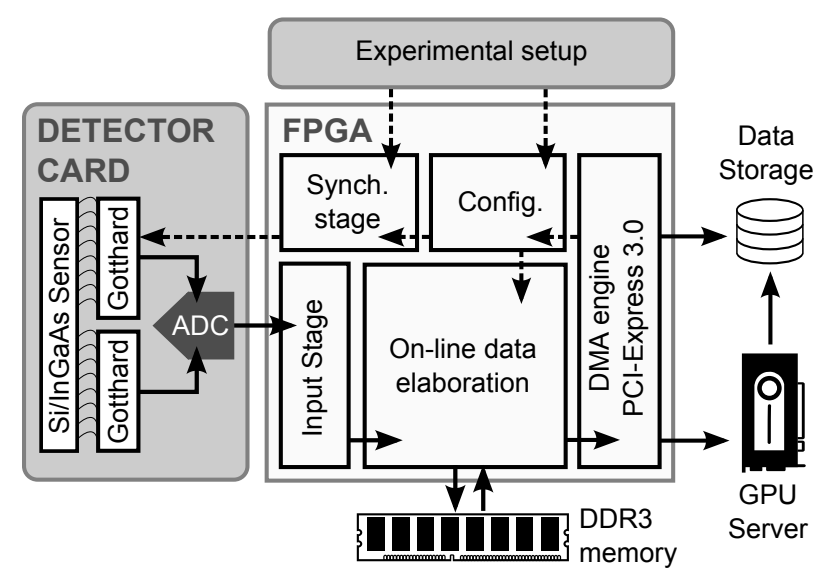

- Data path -... Timing / control signals

Fig. 1. Block diagram of the detector's architecture.

The detector board mounts a single $\mathrm{Si} / \mathrm{InGaAs}$ linear array sensor with 256 pixels and a $50 \mu \mathrm{m}$ pitch. Si and $\mathrm{InGaAs}$ are chosen in order to measure laser pulses in the visible or nearIR spectral domain. Two GOTTHARD 1.6 chips [10] are wire bonded to one sensor. Each channel of the GOTTHARD chip contains a charge-sensitive preamplifier with three different gain settings. An analog multiplexer connects groups of 16 channels to a high-speed analog output buffer. The multiplexer and the analog buffer operate at a maximum switching frequency of $60 \mathrm{MHz}$, therefore limiting the line-rate of the system to $2.7 \mathrm{Mlps}$. The analog outputs are digitized by a commercial Analog-to-Digital Converter (ADC) ${ }^{1}$. Digital-toAnalog Converters (DAC) provide the reference voltages to the GOTTHARD chip. Moreover, a Phase-Locked-Loop (PLL) chip is used to maintain the detector synchronous with the external clock coming from the accelerator timing system.

\footnotetext{
${ }^{1}$ AD9249, 16 channels operating at 65 MSPS with 14-bit resolution
} 


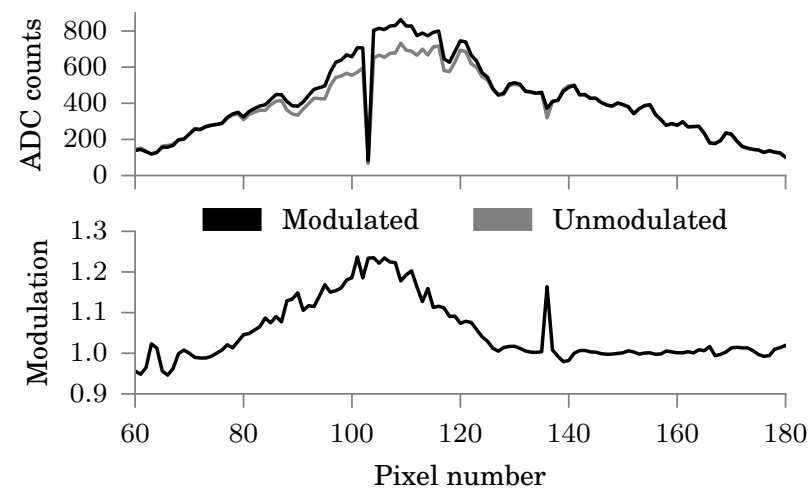

Fig. 2. Measured modulation of the laser pulse. The plot on the top shows a comparison between the signal modulated by the Coulomb field of the electron bunch (black) and the unmodulated one (grey), which is used as reference. The relative modulation is calculated by diving the two signals and is shown in the bottom plot. The optical power of each laser pulse is $12 \mathrm{pJ}$. Note: two pixels (104 and 136) are disconnected from the front-end chip.

The detector board is connected to a custom FPGA-based readout card [11], which controls the operation of the detector. The digital samples from the ADC are acquired and organized in the input stage. An external DDR memory is used as a temporary memory buffer before or after the on-line data elaboration stage. The Direct Memory Access engine described in [12] transfers data to the external DAQ system, with data rates up to $1.4 \mathrm{~GB} / \mathrm{s}$ during continuous acquisition $(16$ bits $\times 256$ pixels@2.7 MHz). The readout card can directly interface external GPU-based computing servers to perform real-time data analysis.

The configuration of the board is programmed by the user through a PCI-Express bank register. Moreover, a dedicated stage is used to synchronize the operation of the board with external system. In particular, two trigger signals are connected to the synchronization stages:

- a fast trigger indicates that a valid laser pulse must be acquired. An internal synchronization stage on the FPGA starts the integration of the signal on the GOTTHARD chip after a programmable delay.

- a slow trigger is generated in the accelerator control room to start the acquisition process. In this way it is possible to synchronize the measurements with other detectors (i.e.: KAPTURE [13]) or with the accelerator machine (to monitor the bunch profile during specific events, i.e.: a beam dump triggered from the control room).

\section{PReliminary MeAsurements AND OUtLOOK}

Preliminary measurements have been carried out at ANKA with the EOSD experimental setup described in [4] and a prototype of the detector board mounting an InGaAs sensor. We used a Yb-doped fiber laser with a programmable repetition rate and with a wavelength of $1050 \mathrm{~nm}$.

The magnitude of the EO signal with respect to the reference laser pulse is shown in Figure 2. The system has been used to monitor the beam conditions over a large number of bunch turns in the storage ring, achieving turn-by-turn resolution with

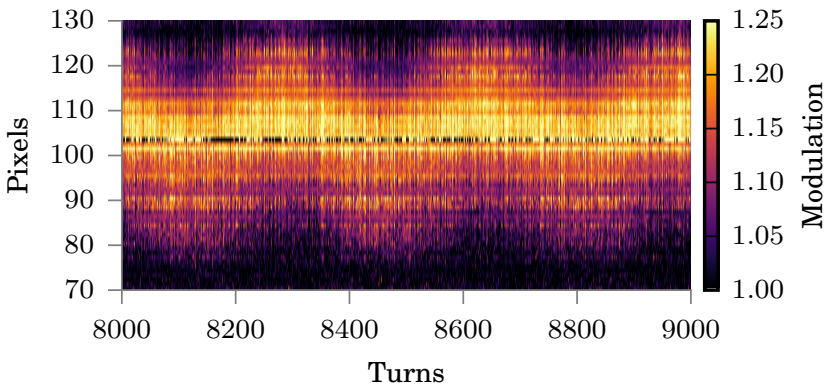

Fig. 3. Intensity of the EOSD signal over 1000 bunch turns (the complete dataset consists of $1 \times 10^{6}$ turns). Every vertical line corresponds to the modulation of a single laser pulse as calculated in Figure 2. The synchrotron oscillation of the electron bunch is clearly visible.

a revolution frequency of $2.7 \mathrm{MHz}$, as shown in Figure 3. The system has been successfully synchronized with other detectors [14] and is currently installed at ANKA. Further test are currently planned to characterize the detector in terms of signal-to-noise ratio.

\section{REFERENCES}

[1] B. Steffen et al., "Electro-optic time profile monitors for femtosecond electron bunches at the soft x-ray free-electron laser FLASH," Physical Review Special Topics - Accelerators and Beams, vol. 12, no. 3, p. 032802, Mar. 2009.

[2] R. Pan et al., "Coulomb field strength measurement by electro-optic spectral decoding system at the CALIFES beam line," Nuclear Instruments and Methods in Physics Research Section A: Accelerators, Spectrometers, Detectors and Associated Equipment, vol. 821, pp. 812, Jun. 2016.

[3] A. Borysenko et al., "Electron bunch shape measurements using electrooptical spectral decoding," Physics Procedia, vol. 77, pp. 3 - 8, 2015, international Conference on Laser Applications at Accelerators, 25-27 March 2015, Mallorca, Spain.

[4] N. Hiller et al., "Status of Single-shot EOSD Measurement at ANKA," international Beam Instrumentation Conference (IBIC), September 2014, Monterey, USA.

[5] I. Wilke et al., "Single-shot electron-beam bunch length measurements," Phys. Rev. Lett., vol. 88, p. 124801, Mar 2002.

[6] U. Happek, A. J. Sievers, and E. B. Blum, "Observation of coherent transition radiation,” Phys. Rev. Lett., vol. 67, pp. 2962-2965, Nov 1991.

[7] Teledyne DALSA Inc. (2016) Piranha4 line camera. [Online]. Available: http://www.teledynedalsa.com/imaging/products/cameras/linescan/piranha4/P4-CM-04K10D/

[8] Sensors Unlimited Inc. (2016) Ingaas high speed linescan camera GL2048-R. [Online]. Available: http://www.sensorsinc.com/products/detail/gl2048-r-ingaas-linescancamera

[9] VITA. (2014) VITA 57 FMC Connectors Datasheet. [Online]. Available: http://www.vita.com/fmc

[10] A. Mozzanica et al., "The GOTTHARD charge integrating readout detector: design and characterization," Journal of Instrumentation, vol. 7, no. 01, p. C01019, 2012.

[11] M. Caselle et al., "High-speed, low-latency readout system with realtime trigger based on GPUs," Real Time Conference, June 2016, Padova, Italy.

[12] L. Rota et al., "A High-throughput Readout Architecture Based on PCIExpress Gen3 and DirectGMA Technology," Journal of Instrumentation, vol. 11, no. 02, p. P02007, 2016.

[13] M. Caselle et al., "An ultra-fast data acquisition system for coherent synchrotron radiation with terahertz detectors," Journal of Instrumentation, vol. 9, no. 1, 2014.

[14] B. Kehrer et al., "Simultaneous detection of longitudinal and transverse bunch signals at ANKA," Proceedings of the International Particle Accelerator Conference (IPAC), May 2016, Busan, Korea. 